\title{
Relationship between the Morphological Character of 5-Nitroacetyl- salicylic Acid Crystals and the Decomposition Rate in a Humid Environment
}

\author{
Makoto Okamura, ${ }^{10)}$ Manabu Hanano, ${ }^{1)}$ and Shoji Awazu ${ }^{1 b)}$ \\ Faculty of Pharmacentical Sciences, the University of Tokyo ${ }^{1}$
}

(Received August 1, 1979)

\begin{abstract}
Two morphologically different crystals, column-shaped and ramified, were sorted under a microscope from a recrystallized batch of 5-nitroacetylsalicylic acid. Their degradation rates in the solid state were quantitatively studied and the progress of decomposition was observed under a polarizing microscope.

Plots of the ratio decomposed against the reaction time were sigmoidal under all reaction conditions, and Avrami's kinetic equation was applicable to this reaction below $40 \%$ degradation. The reactivity of the ramified crystals was greater than that of the column-shaped crystals under all experimental conditions.

Although the parameter $B$ of Avrami's equation, which is assumed theoretically to be proportional to the product of the number of latent reaction nuclei and the voluminal growth rate varied by a factor of $10^{4}$ at maximum under various experimental conditions, the ratio of $B$ between ramified and column-shaped crystals remained fairly constant under all the conditions tested, and was about five.

The results of observation of the decomposing crystals under a polarizing microscope agreed well with Avrami's theory of the formation and growth of reaction nuclei.
\end{abstract}

Keywords- -5-nitroacetylsalicylic acid; morphology of crystals; hydrolysis in solid state; polarized light microscopy; Avrami's theory; chemical kinetics

In the preceding report, ${ }^{2}$ the decomposition of acetylsalicylic acid and its derivatives in the solid state in a humid environment was shown to occur at a constant rate bofore and after the removal of acids produced by hydrolysis during degradation.

This result ruled out a suggested autocatalytic decomposition mechanism involving protons produced in an aqueous layer on the crystal surface. ${ }^{3)}$ A new mechanism is presented in which the reaction regions develop with growing product nuclei at local portions of the crystal surface, based on direct observation of decomposing crystals under a polarizing microscope. An apparently valid rate equation is also presented.

Considerable differences in reaction rate among individual crystals in an experimental batch should be expected, since the decomposition is considered to be occurring heterogeneously in the present mechanism. This kind of difference was actually seen on successive observation of the decomposing crystals under a polarizing microscope, as reported previously.

In this work, the decomposition on exposure to humidity of two morphologically different crystals sorted under a microscope from a recrystallized batch of 5-nitroacetylsalicylic acid (I) was observed with a polarizing microscope, and determined quantitatively by chemical assay. The decomposition rate was found to depend on the morphological character of each crystal. This involves the defects and grain boundaries on the crystals, and may not be related to the arrangement of molecules in the crystal (polymorphism).

The two morphologically different types of crystal are almost the same in crystal size but are probably different in surface area, so that the effect of the area on the reactivity

1) Location: 7-3-1 Hongo, Bunkyo-ku, Tokyo 113, Japan; Present address: a) Department of Pharmacy, College of Science and Technology, Nihon University, Kanda Surugadai, Chiyoda-ku, Tokyo; b) Tokyo College of Pharmacy, 1432-1, Horinouchi, Hachioji-shi, Tokyo.

2) J. Hasegawa, M. Hanano, and S. Awazu, Chem. Pharm. Bull., 23, 86 (1975).

3) L.J. Leeson and A.M. Mattocks, J. Am. Pharm. Assoc., 47, 329 (1958). 
cannot be neglected, though the decomposition reaction area is localized at special regions on the crystals and most of the surface is inactive in the early stage.

As reported previously, ${ }^{2)}$ the decomposition rates of crystals of aspirin as well as I vary with each lot of the crystals. Their decomposition mechanisms were similar, but I is more suitable for kinetic studies because of its high decomposition rate the fact that it does not sublime.

In the present study, I is investigated as a model for aspirin. Avrami's theory concerning the growth of reaction nuclei in solids has often been adapted for kinetic analysis. Equation 1 is Avrami's kinetic equation, which is applicable in comparably early stages of the reaction, ${ }^{4)}$

$$
\alpha / 1-\alpha=B t^{l}
$$

Eq. 1

where $\alpha$ is the ratio decomposed at reaction time $t$, and both $B$ and $l$ are parameters. The parameter $l$ is the dimensional number of the growing nuclei. The parameter $B$ is given by the following equation during the reaction period when the probability of formation of nuclei is less than unity, and when the increase of reaction nuclei can be expressed as an exponential function of time,

$$
B=N_{0} \sigma G^{l} \quad \text { Eq. } 2
$$

where $N_{0}, \sigma$ and $G$ are the concentration of latent nuclei, the shape factor of the nuclei, and the linear velocity of growth, respectively.

The hydrolysis of crystals (I) under humid conditions is presumed to occur by the formation and growth of reaction nuclei as described by Avrami's theory. However, the following points must also be considered. First, the decomposition regions are very locally distributed, probably corresponding to cracks or defects on the crystal surface. Second, large crystals of the decomposition product appear in the later period. Third, stable parts of the crystals (I) selectively remain unchanged even in an almost completely decomposed sample.

Avrami's theory, therefore, requires modification for both the very early stage and the later period (over $40 \%$ decomposed) of decomposition in the present case. In this report, the kinetics of decomposition of the crystals (I) are analyzed in terms of Eq. I, as mentioned above, and the parameter $B$ of the ramified crystals is shown to be several times larger than that of the column-shaped crystals. The kinetic equation can be applied over a wide range of degree of decomposition.

\section{Experimental}

Materials - Crystals of I were synthesized from commercial 5-nitrosalicylic acid by the method of

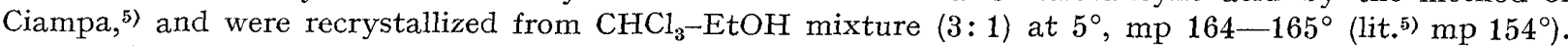
Anal. Calcd for $\mathrm{C}_{9} \mathrm{H}_{7} \mathrm{NO}_{6}: \mathrm{C}, 48.00 ; \mathrm{H}, 3.13 ; \mathrm{N}, 6.17$. Found: $\mathrm{C}, 48.01 ; \mathrm{H}, 3.13 ; \mathrm{N}, 6.22$. All other chemicals used were guaranteed grade commercial materials and were not subjected to further purification.

Morphological Sorting of Crystals (I) C Crystals (I) which had been sieved (80-150 mesh) without grinding were sorted into two morphologically different forms under a microscope from a recrystallized batch of I. One (column-shaped crystals) had a smooth surface, while the other (ramified crystals) had on irregular surface and branches as shown in Fig. 1.

Experimental Procedure and Chemical Assay_-A given amount of crystals (I) was placed in reaction vessels as described in the preceding report with the inorganic salts listed in Table I for humidity control, decomposed in a water bath at 80 or $70^{\circ} \pm 0.1^{\circ}$ for an appropriate time, and then dissolved in a mixture of $20 \mathrm{ml}$ of $\mathrm{CHCl}_{3}-\mathrm{EtOH}(1: 2)$ mixture and $1 \mathrm{ml}$ of $1 \% \mathrm{FeCl}_{3}$ in $0.1 \mathrm{~N} \mathrm{HCl}$ aqueous solution for colorimetric determination with a Hitachi 124 spectrophotometer (498 $\mathrm{nm}$ absorption wavelength) as described previously.

Polarized Microscopic Observation-Crystals (I) dispersed on a small slide glass were reacted as described above. Photographs of the reacted crystals were taken under a polarized microscope through crossed nicols and a gypsum test plate.

4) M. Avrami, J. Chem. Phys., 7, 1103 (1939); idem, ibid., 8, 212 (1940); idem, ibid., 9, 177 (1941).

5) G. Ciampa, Ann. Chim. (Rome), 54, 975 (1964). 


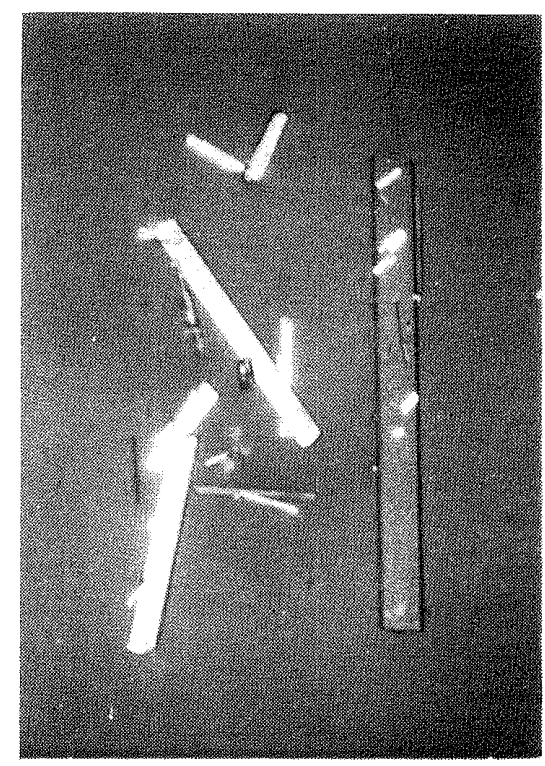

a)

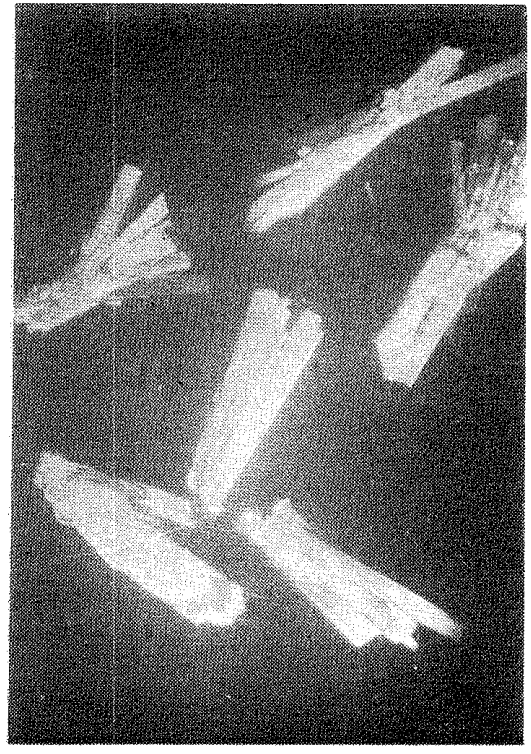

b)

Fig. 1. Microscopic Photographs $(\times 100)$ of Morphologically Different Crystals
a) Column-shaped crystals.
b) Ramified crystals.

TABLE I. List of Inorganic Salts used for Humidity Control

\begin{tabular}{lcccccc} 
Temperature $\left({ }^{\circ} \mathrm{C}\right)$ & $\overbrace{}^{2}$ & & \multicolumn{3}{c}{$80^{\circ}$} \\
Relative humidity $(\%)$ & 80 & 22 & 79 & 50 & 23 \\
Saturated salt system & $\mathrm{KCl}$ & $\mathrm{KF}$ & $\mathrm{KCl}$ & $\mathrm{NaBr}$ & $\mathrm{KF}$ \\
\hline
\end{tabular}

\section{Results and Discussion}

The plots of the ratio decomposed against the reaction time under various experimental conditions of relative humidity and temperature are shown in Figs. 2-5. The time courses of decomposition were sigmoidal, having an induction period with a very slow decomposition rate, an acceleration period in the early stage of the reaction, and a deceleration period towards the final stage.

The decomposition of the ramified crystals was faster and accelerated more quickly than that of the column-shaped crystals under all conditions tested, showing the influence of morphological character. Since plot of the ratio decomposed against time was nearly linear, the steepest slope (dotted line in the figures) was used to calculate the maximal decomposition velocity, and the apparent induction period was calculated from the intercept.

The relationships between the relative humidity in the reaction vessel and the apparent induction period and the maximal decomposition velocity are shown in Figs. 6 and 7 , respectively. The induction period decreased with increase in humidity, while the maximal decomposition velocity increased, and the reactivity of the ramified crystals (in terms of both induction period and maximal velocity) was greater than that of the column-shaped crystals under all the experimental conditions tested.

Eq. 1 can be converted into the following form.

$$
\log (\alpha / 1-\alpha)=\log B+l \log t \quad \text { Eq. } 3
$$

Plots based on this equation are illustrated in Figs. $8-10$ at 70 and $80^{\circ}$. It is clear that Eq. 1 is valid up to $40 \%$ decomposition. In the reaction in $23 \%$ humidity at 70 and $80^{\circ}$ 


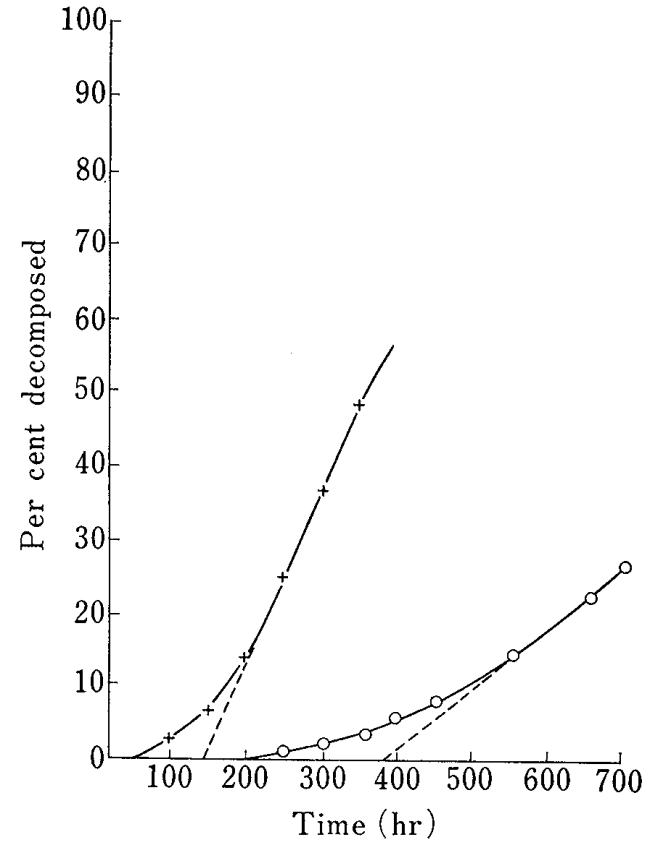

Fig. 2. Time Courses of Decomposition of Column-Shaped Crystals at $70^{\circ}$

Open circles and crosses indicate 22 and $80 \%$ humidity, respectively. The solid line was calculated from Avrami's equation and the dotted line shows the maximal decomposition velocity.

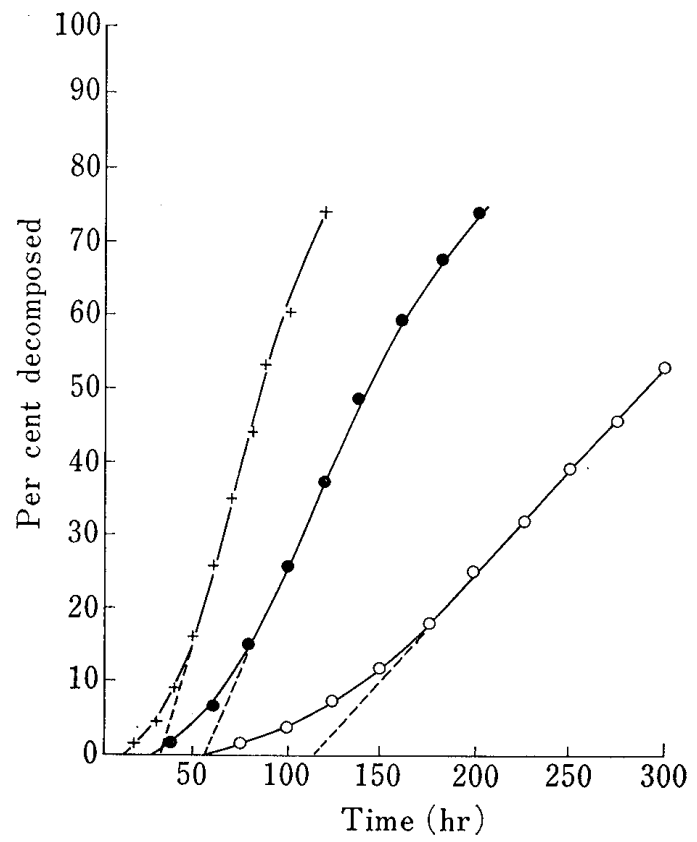

Fig. 4. Time Courses of Decomposition of Column-shaped Crystals at $80^{\circ}$

Open circles, closed circles, and crosses indicate 23,50 , and $79 \%$ humidity, respectively. The solid line is calculated from Avrami's equation and the dotted line shows the maximal fractional decomposition velocity.

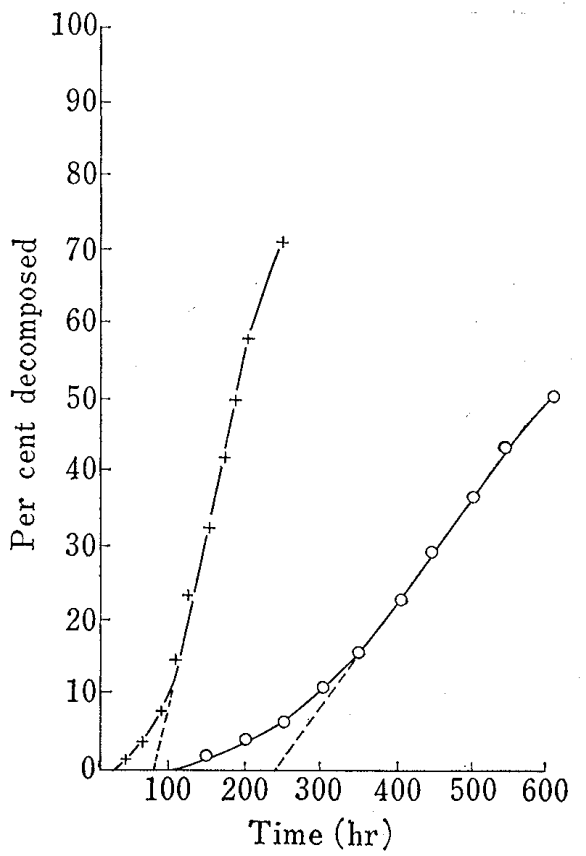

Fig. 3. Time Courses of Decomposition of Ramified Crystals at $70^{\circ}$

Symbols: see Fig. 2.

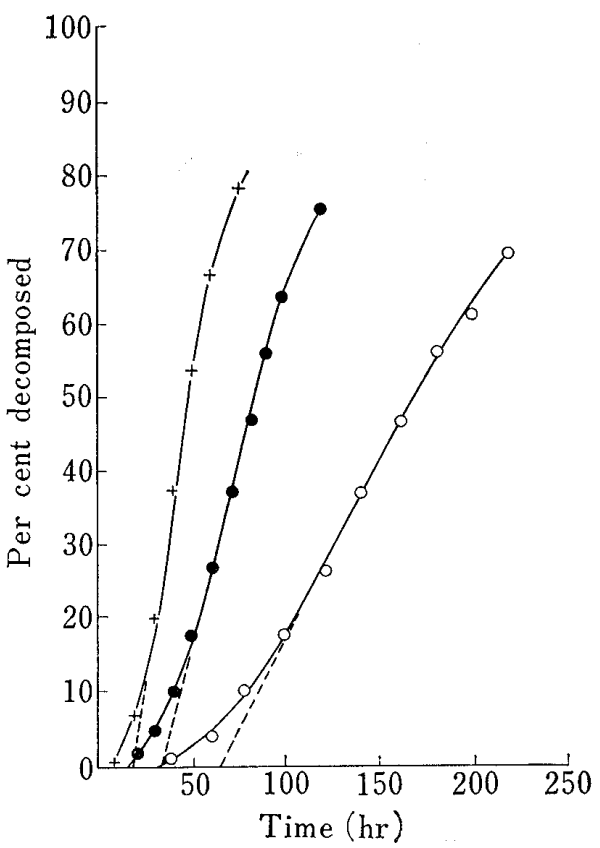

Fig. 5. Time Courses of Decomposition of Ramified Crystals at $80^{\circ}$

Symbols: see Fig. 4 . 
(slow decomposition) the plot diverges from the straight line in the early stage of decomposition. This may be a result of the existence of localized reaction regions on the crystal surface. The rapid decrease in the reaction rate from the calculated values over $40 \%$ decomposition may arise not only from the overlapping of reaction regions, as pointed out by Avrami, but also from the inertness of remaining stable portions of crystals.

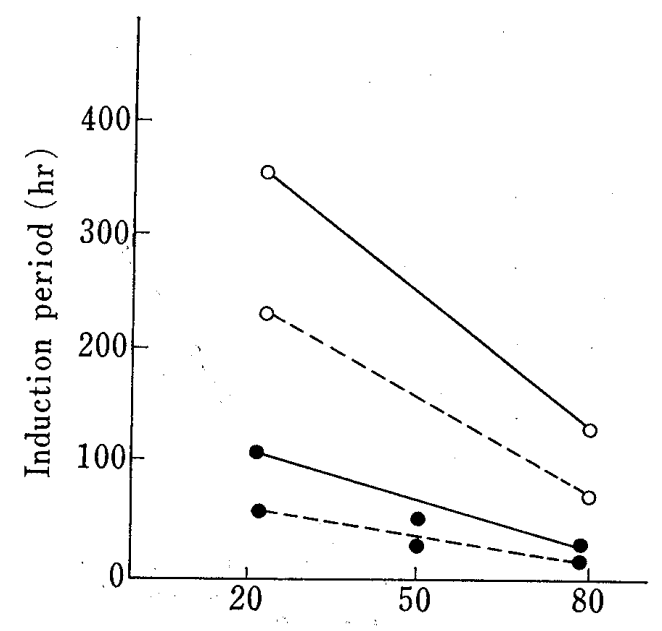

Relative humidity (\%)

Fig. 6. Relationship between Induction Period of Decomposition and Relative Humidity

Solid and dotted lines are for column-shaped and ramified crystals, respectively. Open and closed circles are at $70^{\circ}$ and $80^{\circ}$, respectively.

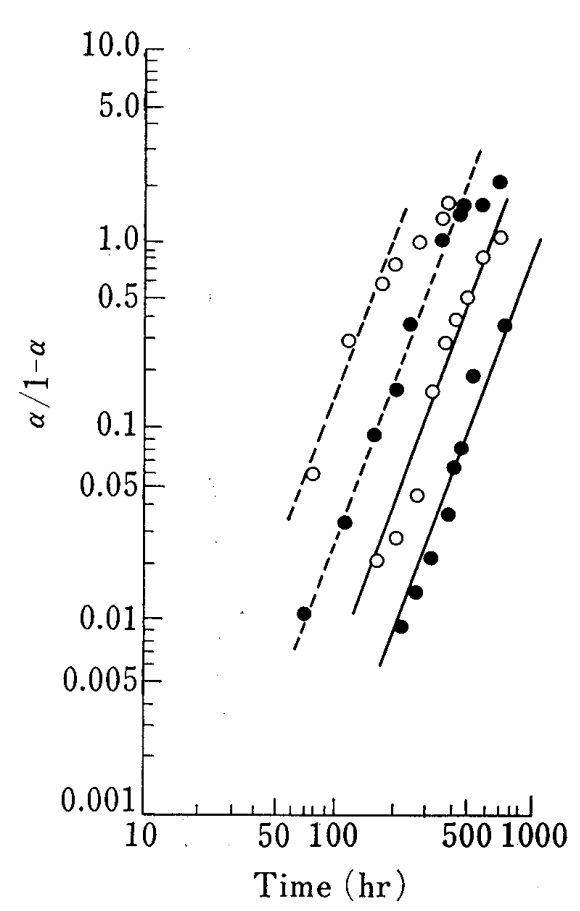

Fig. 8. Kinetic Plot based on Avrami's Equation for the Decomposition at $70^{\circ}$

Open and closed circles are for ramified and column-shaped crystals, respectively. Solid and dotted lines are in 22 and $80 \%$ humidity, respectively, $\alpha$ is the decomposition ratio.

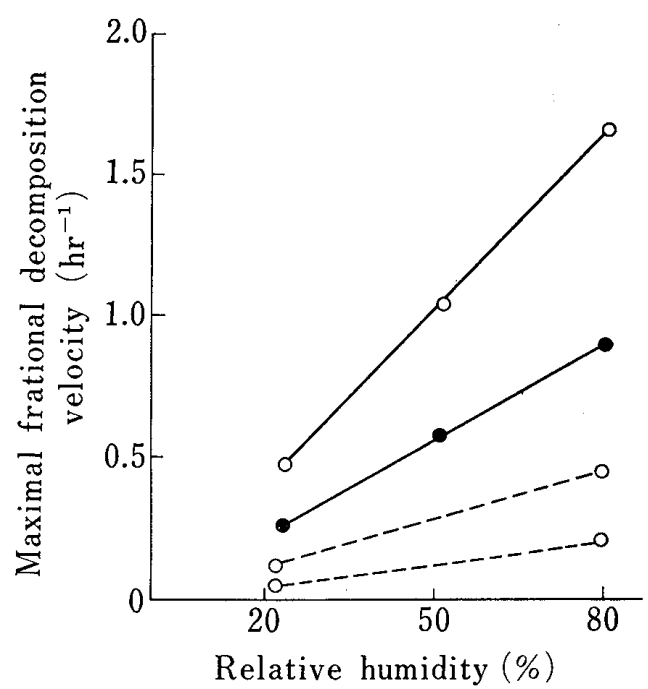

Fig. 7. Relationship between Maximal Fractional Decomposition Velocity and Relative Humidity

Symbols: see Fig. 6.

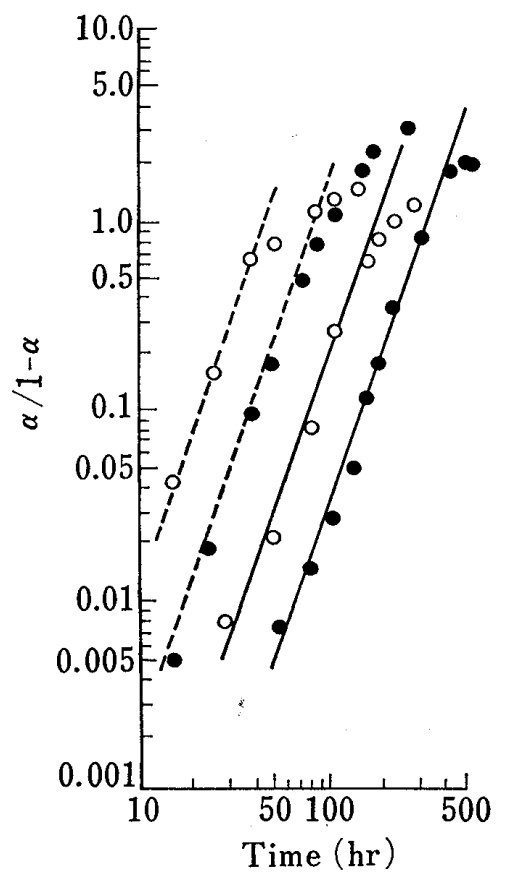

Fig. 9, Kinetic Plot based on Avrami's Equation for the Decomposition at $80^{\circ}$

Open and closed circles are for ramified and column-shaped crystals, respectively. Solid and dotted lines are in $\mathbf{2 3}$ and $79 \%$ himidity, respectively, $\alpha$ is the decomposition ratio. 
Table II presents the parameters $B$ and $l$, calculated from Figs. 8-10. The values of $l$ are all between 2.4 and 3.5, and hence the growth of reaction nuclei is presumed to be three-dimensional. The median decomposition was about $12 \%$ throughout the experiments.

From these results, the values of $B$ for comparison among various conditions and between the crystal shapes were recalculated for a decomposition of $12 \%$ and assuming the value of $l$ to be 3 , as follows,

$$
\log B=\log (0.12 / 0.88)-3 \log t_{\alpha=0.12} \quad \text { Eq. } 4
$$

where $t_{a=0.12}$ is the time of $12 \%$ decomposition read from the straight line. The solid lines in Figs. 2-5 and the straight lines in Figs. 8-10 represent the recalculated results, and are clearly in reasonable accord with the observed data.

As shown in table II, while $B$ varied by a factor of $10^{4}$ at maximum, the ratio of the $B$ values, $\beta=B_{\mathrm{b}} / B_{\mathrm{a}}$, for the ramified crystals $\left(B_{\mathrm{b}}\right)$ and the column-shaped crystals $\left(B_{\mathrm{a}}\right)$ was hardly affected by the humidity at $80^{\circ}$, though it was somewhat affected by humidity at $70^{\circ}$.

This suggests strongly that the decomposition of the two morphologically different crystals proceeds by the same mechanism under all the conditions tested. From Eq. 2, the difference of $B$ value between two kinds of crystals under given reaction conditions depends on the concentration of latent nuclei and the velocity of nuclei growth if the shape factor is the same.

TABle II. Pararicter Values, $\beta$ and $l$, of Avrami's Kinetic Equation under Various Decomposition Conditions

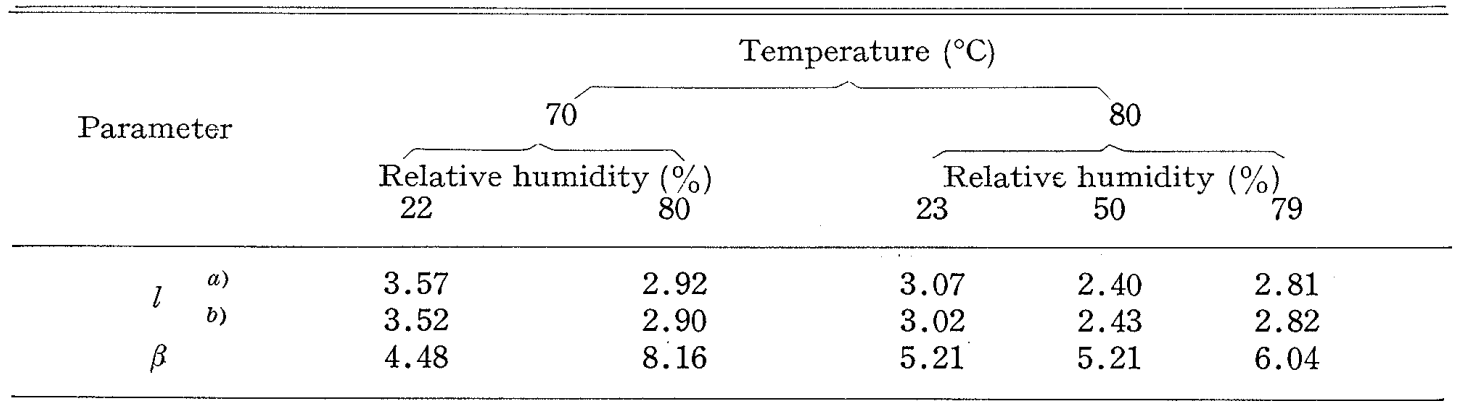

a) Column-shaped crystals.

b) Ramified crystals.

When the decomposition of crystal (I) is observed under a polarized microscope, 5 -nitrosalicylic acid formed by the decomposition of I presumably appears as microcrystals with random orientation, and is observed as dark regions on the original crystals. Fig. 11 shows photographs of crystals partially decomposed in $79 \%$ humidity at $80^{\circ}$.

In the early stage of decomposition, the reaction regions, appearing as dark spots, are scattered on the surface of the column-shaped crystals, but are concentrated at the forks 


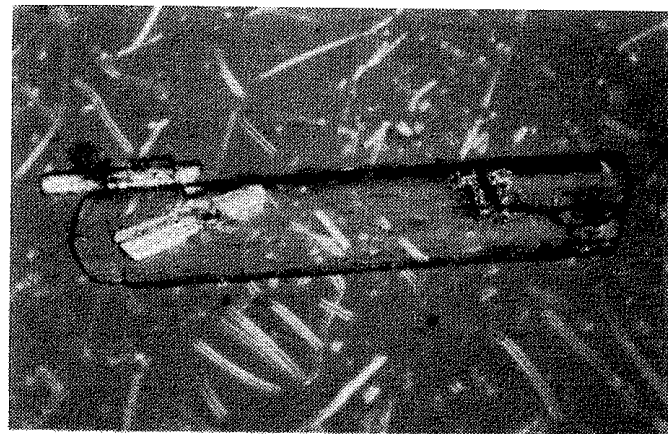

a)

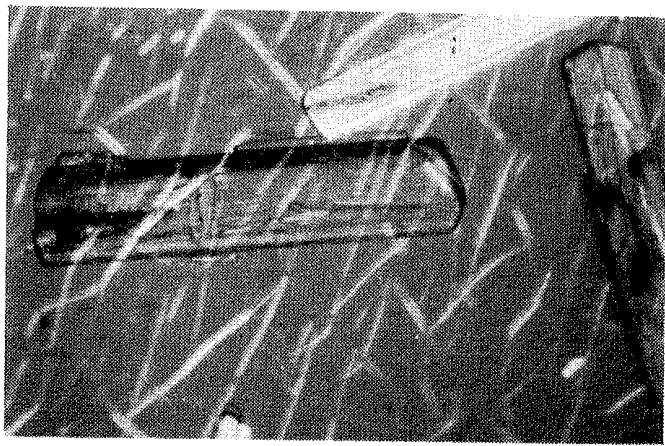

b)

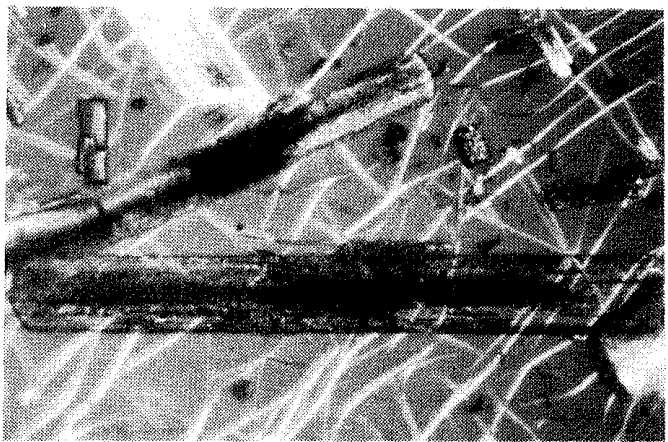

c)

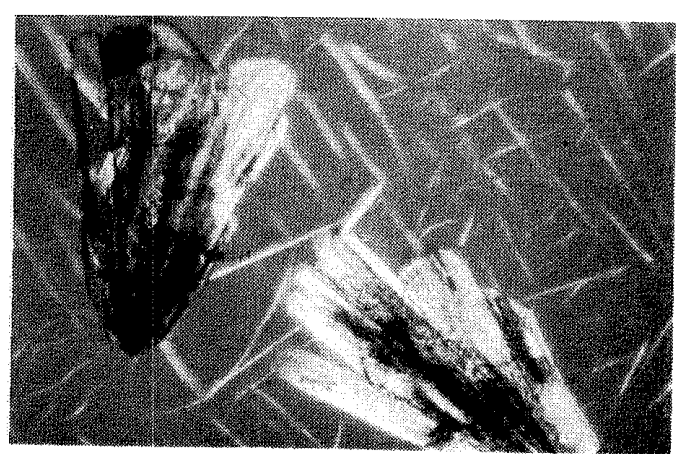

d)

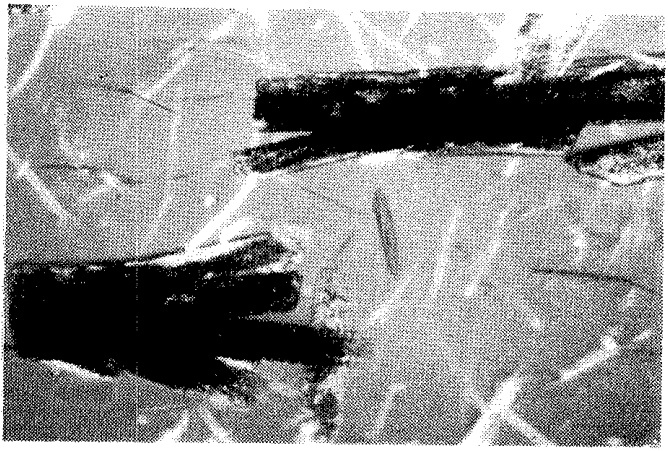

e)

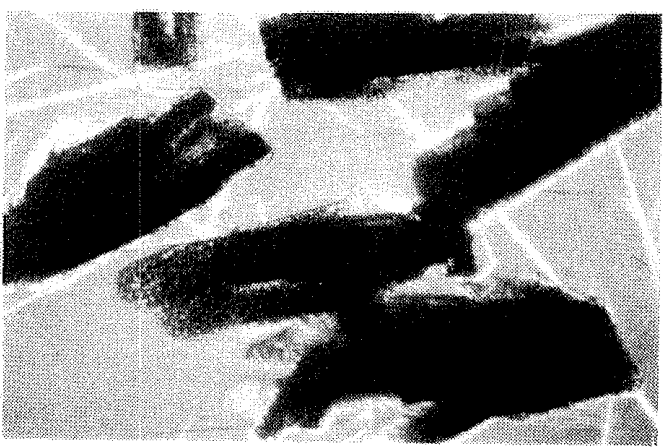

f)

Fig. 11. Polarized Light Microscopic Photographs $(\times 100)$ of Partially Decomposed Crystals at Various Reaction Times in $79 \%$ Humidity at $80^{\circ}$

$$
\begin{aligned}
& \text { a), b), c): column-shaped crystals. } \\
& \text { d), e), f): ramified crystals. } \\
& \begin{array}{lcccccr} 
& \text { a) } & \text { b) } & \text { c) } & \text { d) } & \text { e) } & \text { f) } \\
\text { Reaction time (hr) } & 16 & 25 & 40 & 16 & 25 & 40
\end{array} \\
& \begin{array}{lllllll}
\text { Per cent decomposed } & 0.5 & 2.0 & 8.9 & \mathbf{4 . 3} & \mathbf{1 4 . 6} & 36.9
\end{array}
\end{aligned}
$$

of ramified crystals. The regions progressively spread out on the crystal surface and cover the whole surface at about $30 \%$ decomposition.

The high reactivity at the fork might be caused by defects of the crystal lattice, though moisture condensation at the fork cannot be excluded as a possible cause. Defects on the column-shaped crystal surface were also observed to bear reaction regions with high probability. Overall, these observations are consistent with the growth and birth of reaction nuclei assumed by Avrami's theory.

The results at $80^{\circ}$ were in good accord with those at $70^{\circ}$. 